\title{
Signed Graph and its Balance Theory in Transportation Problem
}

\author{
Arun Kumar Baruah \\ Department of Mathematics \\ Dibrugarh University \\ Dibrugarh786004, Assam,India
}

\author{
Manoshi Kotoky \\ Department of Mathematics \\ Dibrugarh University \\ Dibrugarh786004, Assam,India
}

\begin{abstract}
Signed graph can be used as a graph theoretic tool to study transportation problem. A transportation problem can be efficiently modeled as a graph where the nodes represent the destinations and the edges represent the relationship among them. With the help of signed graph the relationship between various destinations in a transportation network can be represented. The simplest approach to study such a group of destinations is to draw a graph in which the destinations are the nodes (or vertices) and there is an edge joining each pair of destinations who are related in some way. It can also be checked whether the graph is stable or unstable with the use of signed graph and balance theory.
\end{abstract}

\section{Keywords}

Signed graph, balanced theory, transport network, partitionable and non partitionable signed graphs.

\section{INTRODUCTION}

A Transportation problem is one of the earliest and most important applications of linear programming problem. Description of a classical transportation problem can be given as follows-A certain amount of homogeneous commodity is available at number of sources and a fixed amount is required to meet the demand at each number of destinations. A balanced condition (i.e. Total demand is equal to total supply) is assumed. Then finding an optimal schedule of shipment of the commodity with the satisfaction of demands at each destination is the main goal of the problem. In 1941 Hitchcock [1] developed the basic transportation problem along with the constructive method of solution and later in 1949 Koopmans [2] discussed the problem in detail. Again in 1951 Dantzig [3] formulated the transportation problem as linear programming problem and also provided the solution method. Now a day's transportation problem has become a standard application for industrial organizations having several manufacturing units, warehouses and distribution centers.

In the first article on signed graphs - graphs where edges are labeled positive or negative (Harary, F., [4]) to treat a question in social psychology [5] giving a simple characterization of those in which the product of signs around every cycle is positive (such graph is called balanced). Later Beineke and Harary [6] included signed vertices and asked the analogus question of characterizing the vertex - signed graphs around every cycle are positive. Also preliminary results may found by Acharya [7] and Rao [8], Hoede [9]. Signed graph and balanced theory are most important part of the graph theory.

\section{SIGNED GRAPH}

A signed graph (Fig 1) is simply a graph where each edge between the nodes is labeled as positive (+) or negative (-).
A signed graph $G$, or briefly an $s$-graph consist of a set $E$ of n point $P_{1}, P_{2}, \ldots, P_{n}$ together with two disjoint subsets $L^{+}, L^{-}$of the set of all unordered pairs of distinct points. The elements of the sets $L^{+}, L^{-}$are called positive lines and negative lines respectively.

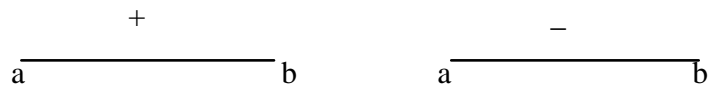

Fig 1: Signed Graph

The concept of signed graph is used if the relationship between each pair of nodes is symmetric. With the help of signed graph, the interpersonal relationships between groups of individuals can be represented. The simplest approach to study such a group of individuals is to draw a graph in which the individuals are the nodes and in which there is an edge for ' $x$ ' to node ' $y$ ' if $x$ is in some relation to $y$. This relationship may be like or dislike, associate with or avoids and so on. Two different relationship can be included in a graph by using two different signs i.e. positive $(+)$ and negative $(-)$ to distinguish them. Then the presence of an edge means that there is a relationship between the nodes and the indication of a (+) sign represents a positive relation such as like, agree etc. and the $(-)$ sign represents the other relation such as dislike, disagree, hate etc. which lead to the existence of signed graph.

\section{BALANCED AND UNBALANCED SIGNED GRAPH}

A graph is said to be balanced (or stable) if all the cycles of the signed graph are positive $(+)$ or there is a question that 'Is it possible to partition vertices of a signed graph, so that every line that connects vertices that belong to the same cluster is positive and every line that connects two vertices that belongs to different clusters is negative?'

If it is possible to partition vertices in this way, we call the signed graph partitionable or clusterable. Especially important are signed graphs where vertices can be partitioned into two clusters. Such graphs are called balanced.

Similarly, a graph is called unbalanced if there exists at least one cycle which is negative (-).

The following theorems may be cited in this article.

\subsection{Theorem}

A Signed graph is balanced if and only if for each pair of distinct pair of points $U$ and $V$, all the paths joining $U$ and $V$ have the same sign [10].

\subsection{Theorem}

A signed graph $G$ is called balanced if and only if its vertex set $V$ can be partitioned into two disjoint subsets namely $V_{l}$ and $V_{2}$, in such a way that each positive line of $G$ joints two 
points of the same subset and each negative line joints two points of different subsets [10].

\subsection{Theorem}

Every semi cycle is positive [11].

\section{BALANCE THEORY AND APPLICATION}

In parallel to the classical graph theory there is an emerging theory of signed graphs, graphs where each edge receives a positive or a negative sign. By introducing signs into a graph, all its cycles become either balanced or unbalanced according to whether the sign product is positive or negative, respectively.

To illustrate the concept of balance theory of signed graph let us consider a social problem where friendliness or unfriendliness occurs between certain pairs of individuals
[11].Assume there is a rumor which has two basic forms, one true and one false. Suppose that anyone would tell the rumor to a friend in the same form he had received it, but would change the form if he were pass the rumor to someone with whom he is unfriendly. If the system is balanced, each person will hear only one version of the rumour regardless of how it reached him (Theorem 3.1-all the paths have same sign); also the person who started the rumor will hear it returned to him in the same form as he originally knew it (Theorem 3.3-every semi cycle is positive). The two theorems mentioned so far allow partitioning of a signed graph into two clusters. In the next figure all possible signed graphs on three vertices are shown. According to balance theorems the top four graphs in Fig 2 are balanced and the bottom four in Fig $\mathbf{3}$ are unbalanced [5].
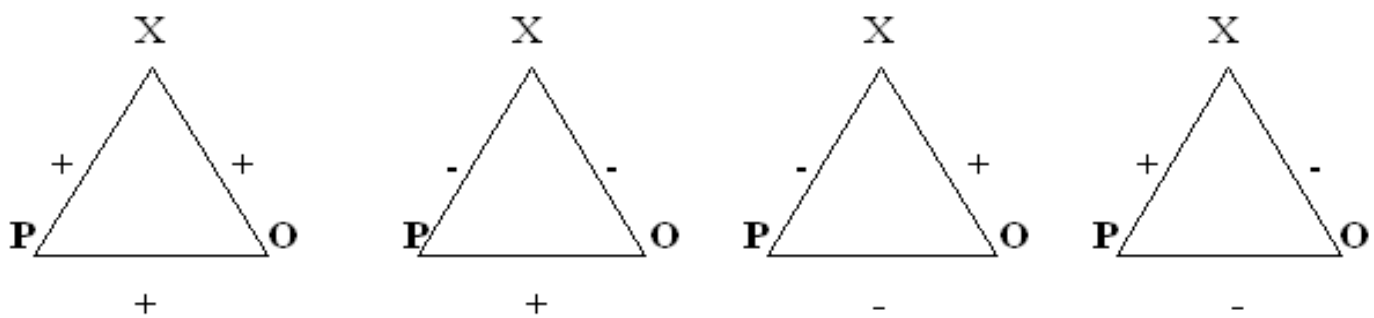

Fig 2 : Four Balanced Triadic Configurations
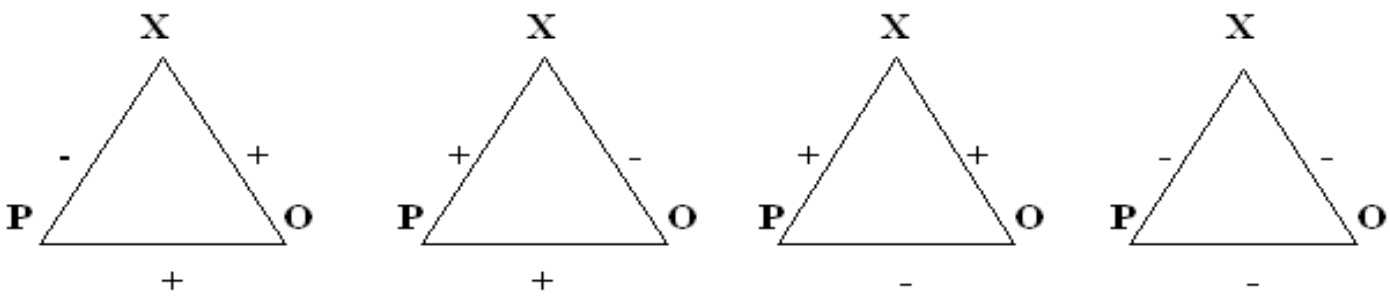

Fig 3 : Four Unbalanced Triadic Configurations

\section{The four situations of Fig 1 can be explained as follows:}

1. A friend of my friend is also my friend.

2. An enemy of my friend is my enemy.

3. A friend of my enemy is also my enemy.

4. An enemy of my enemy is my friend.

The first three situations are clear, but somebody would maybe prefer the statement: An enemy of my enemy is also my enemy, instead of the last statement. This situation is shown in the last graph in Fig 3.

\section{FORMULATION OF THE PROBLEM}

A transportation problem can be modeled by a graph where the nodes represent the various destinations and the edges represent the relationship among them. Thus transportation control problem results in a complete graph i.e. every node is connected to every other node by an edge with a sign associated with it.

Signed graph representation enables the study of complex structures of inter related quantities and offers a graph approach which is both descriptive as well as predictive to study transportation problem

Here a transportation problem for symmetric case at seven destinations is considered, where $a, b, c, d, e, f, g$ are the destinations. Here two cases will be shown, one for the balanced situation and another for unbalanced situation as shown below:

Case-I: Representing a balance of transportation problem by using signed graph. The relationship of the destinations is shown in Fig 4. 


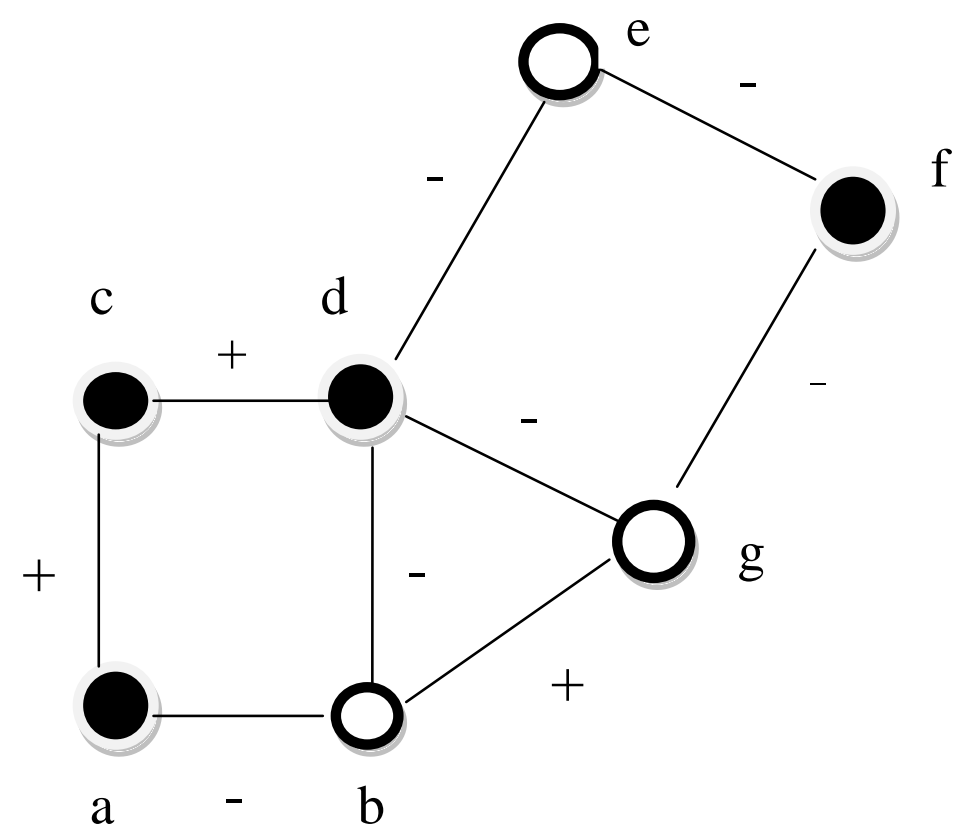

Fig 4: Balanced Network

Here, vertices represent the various destinations and the edges joining the vertices represent a relationship between the vertices (destinations). The positive $(+)$ sign indicates that there is an allowable path between the destinations, and the negative (-) sign represent that there is no such path between the destinations. Therefore, from the Theorem 3.1 and Theorem 3.2, it is found that the system represented by Fig 4 is a balanced. As vertex set can be partitioned into two disjoint sub-sets and edges joining the vertices of the same sub set are positive and the edges joining the vertices of two different sub-sets are negative. Here the vertices $a, c, d, f$ can be put in one set as $M=\{a, c, d, f\}$, and the vertices $b, e, g$ can be put another set as $N=\{b, e, g\}$, such that every edges between $M=\{a, c, d, f\}$, is positive and the edges from the vertex set $N=\{b, e, g\}$, to any vertex of $M=\{a, c, d, f\}$, are negative Hence, it is balanced.

Case-II: Representing an unbalance transportation problem by using signed graph. The relationship of the destinations is shown in Fig 5.

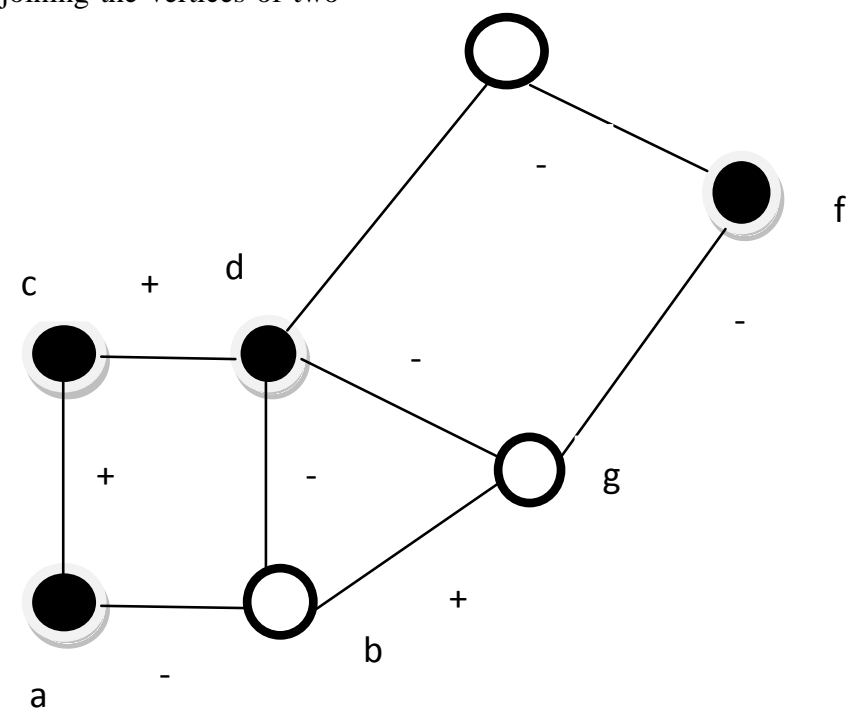

Fig 5: Unbalanced Network

The above signed graph in Fig $\mathbf{5}$ is unbalanced. Here the set of vertices (i.e. destinations) cannot be portioned into two disjoint subsets, satisfying the conditions of the Theorem 3.1 and Theorem 3.2. Also there are two negative cycles which mean that the graph representing the above transportation problem is unbalanced.

From the above discussion it is clear that the transportation problem at a balanced situation is smooth, efficient that is it is easier to transport various objects. On the other hand the transportation problem at an unbalanced situation is less efficient as compared to the balanced situation in the transportation of objects.

\section{CONCLUSION}

To illustrate the balance theory of signed graph, an example for two cases is considered, one for the balanced case and the other for the unbalanced case of a transportation problem. If the graph is balanced, smooth transportation of commodity is possible and otherwise not. Though here comparatively less 
number of destinations is considered, however the procedure can be generalized and can be applied to large number of destinations and to be checked whether transportation of commodity among the destinations are efficient or not. In future the balance theory can be more efficiently used in various fields.

\section{REFERENCES}

[1] Hitchcock, F.L., The distribution of a product from several sources to numerous localities, Journal of Mathematical Physics, vol 20, (2006), pp. 224-230.

[2] Koopmans T. C., Optimum Utiliztion of Transportation System, Econometrica, Supplement vol 17, (1949).

[3] Dantzig, G.B., Linear Programming and Extensions, Princeton University Press, Princeton, N J, (1963).

[4] Harary, F, "On the notion of balance of a signed graph", Michigan Math. J. 2, (1954), 143-146.

[5] Cartwright, D., and Harary, F., "Structural balance: a generalisation of Heider's theory". (1956), Psychological Rev. 63, 277-293.
[6] Beineke, W., Harary, F., and Lowell, "Consistent graphs with signed points." Riv. Mat. Sci. Econom. Social, (1978), 81-88.

[7] Acharya,B.D. and B. Devadas, B., " A characterization of consistent marked graphs" Nat. Acad. Sci. Letters (India) , (1983), 431-440.

[8] Rao, S.B., "Characterizations of harmonious marked graphs and consistent nets". J. Combin. Inform System Sci. 9, (1984), 97-112.

[9] Hoede C., A characterization of consistent marked graphs, J. Graph Theory, 16 (1992): pp.17- 23.

[10] Roberts F.S, Graph Theory and Its Application to Social Science, Regional Conference Series in Applied Mathematics, (1978).

[11] Chartrand, G., Introductoty Graph Theory. Chapter 8: Graphs and Social, New York: Dover Publications, Inc, (1985): 171-182. 